\title{
EL SURGIMIENTO DEL MOVICE Y LA TEORÍA CONTEMPORÁNEA DE LOS MOVIMIENTOS SOCIALES
}

Agustín Eugenio Martínez Elías

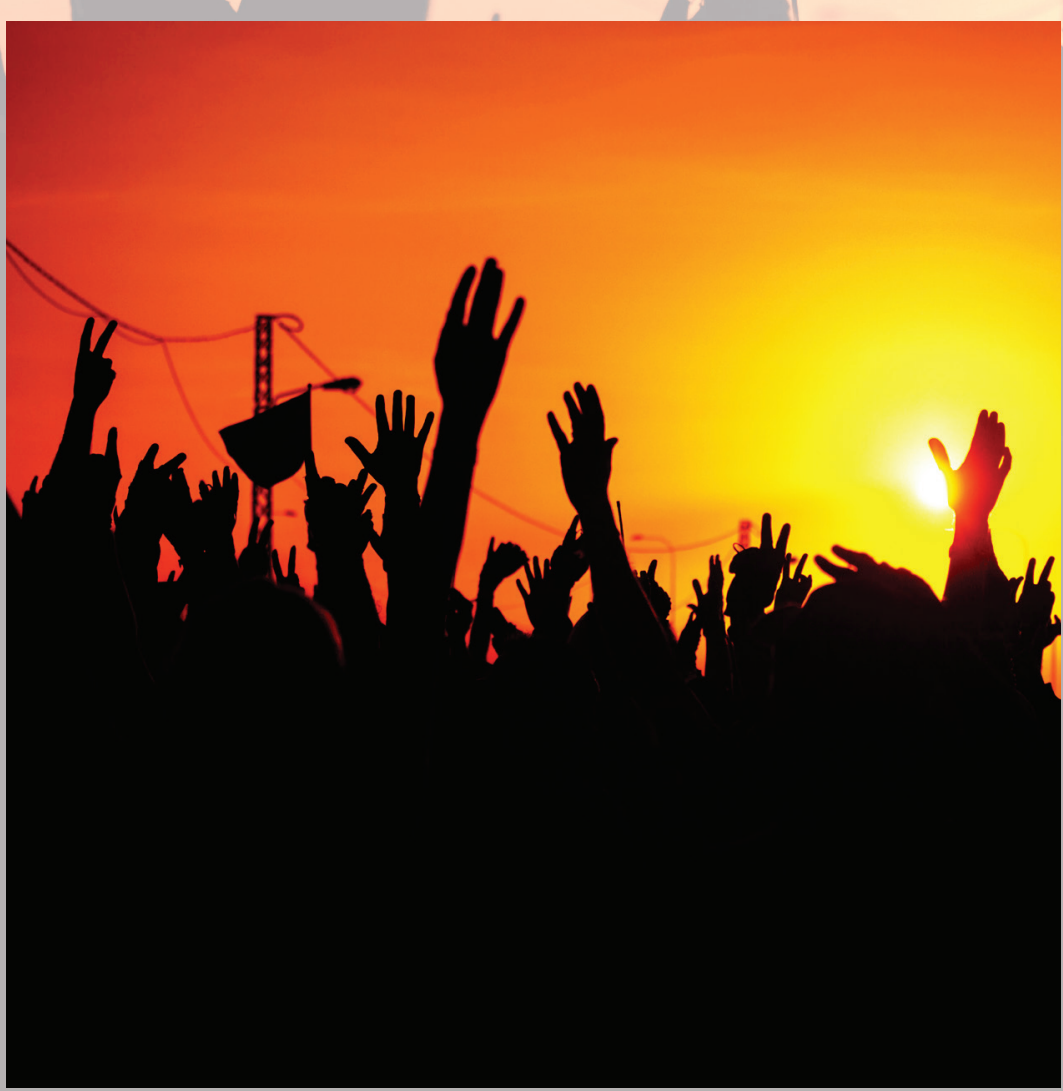





\title{
EL SURGIMIENTO DEL MOVICE Y LA TEORÍA CONTEMPORÁNEA DE LOS MOVIMIENTOS SOCIALES
}

\author{
Agustín Eugenio Martínez Elías \\ Universidad Autónoma de Zacatecas
}

\section{Resumen}

El Movimiento nacional de víctimas de crímenes del Estado (Movice) surge para encabezar una lucha en contra de la impunidad de la violencia generada por el Estado colombiano, por medio de la construcción de una visión propia y clara del origen y de los alcances del conflicto y de la violencia nacional, por una parte y, por otra, de una articulación transversal de diversos movimientos sociales y colectivos que sumaron esfuerzos. En este artículo se analiza el surgimiento histórico del Movice a la luz de las teorías contemporáneas de los movimientos sociales y se concluye con la identificación de algunas fortalezas y áreas de mejora en esta lucha por la verdad, la justicia, la reparación y las garantías de no repetición.

Palabras clave: movimientos sociales, víctimas, derechos humanos, impunidad.

El autor: C argo, filiación. Correo electrónico: elias.martz140@gmail.com

Recibido: 27 de junio de 2017; evaluado: 1 de septiembre de 2017; aceptado: 1 de octubre de 2017 


\title{
THE EMERGENCE OF MOVICE AND THE CONTEMPORARY THEORY OF SOCIAL MOVEMENTS
}

\author{
Agustín Eugenio Martínez Elías \\ Universidad Autónoma de Zacatecas
}

\begin{abstract}
The National Movement of Victims of State Crimes has led the struggle against the impunity of the victims of violence generated by the Colombian State, through the construction of a clear vision of the origin and scope of the conflict and national violence, and through a transversal articulation of different pre-existing social and collective movements that joined efforts. This article analyzes the historical emergence of the movement in the light of contemporary theories of social movements, concluding with the identification of some of its strengths and areas of improvement in this struggle for truth, justice, reparation and guarantees of non-repetition.
\end{abstract}

Keywords: Social movements, victims, human rights, impunity.

About the author: Attorney from Universidad Autónoma de Zacatecas.

Received: June 27, 2017; evaluated: September 1, 2017; accepted: October 1, 2017 


\section{Introducción}

Diversos acuerdos de paz y desmovilización que ha celebrado el Estado colombiano con agrupaciones armadas han puesto en el centro del debate los derechos de las víctimas y los medios por los cuales ellas han de exigirlos frente a una inminente negociación.

En este contexto, las víctimas de crímenes cometidos por el Estado ocupan un lugar especial en el proceso de lucha por los derechos, ya que el ente encargado de respetarlos, protegerlos, promoverlos y garantizarlos es el que los ha violentado. El Movimiento nacional de víctimas de crímenes del Estado surge en Colombia como un movimiento social que busca encaminar los esfuerzos de la lucha en contra de la impunidad de tales crímenes; en primer término, hace frente a la entrada en vigor del marco de justicia y paz en el año 2005, con el que se pretendía dar tratamiento jurídico a los mismos y que, desde la perspectiva de muchas de las víctimas, era una franca vulneración a su dignidad.

Hasta el momento, los enfoques de análisis de la lucha por los derechos de las víctimas se han centrado en investigaciones dogmáticas del derecho; pocas se orientan a estudiarla desde una aproximación sociológica.

Este artículo busca esbozar, a partir de la postura de diversos teóricos de los movimientos sociales, el surgimiento, las fortalezas y las debilidades del Movimiento nacional de víctimas de crímenes del Estado frente a su lucha por la justicia, la verdad, la reparación y las garantías de no repetición.

Comienza con un breve recuento histórico de su origen y su visión de la violencia en el país y luego delinea algunos elementos teóricos explicativos del principio y éxito de los movimientos sociales, como la estructura de oportunidades políticas, la forma organizacional del movimiento, el proceso de encuadre de la realidad ejercida por el movimiento y el repertorio de acción colectiva. Por último, a la luz de esos elementos teóricos, se analizan las fortalezas y debilidades del Movice como un movimiento social organizado en torno a la lucha por los derechos humanos.

\section{Metodología}

Esta es una investigación sociojurídica en la que se han utilizado los métodos de investigación analítico, deductivo e histórico y las técnicas de investigación 
de consulta y análisis de fuentes documentales sobre el Movimiento nacional de víctimas de crímenes del Estado y de diversos teóricos de los movimientos sociales.

\section{El Movimiento nacional de víctimas de crímenes del Estado}

El Movimiento nacional de víctimas de crímenes del Estado (Movice) es un movimiento social que lucha en contra de la impunidad de los crímenes cometidos por el Estado y por grupos paramilitares colombianos.

Este movimiento surgió en 2005, con el afán de que no sean invisibilizados los casos de desaparición forzada, ejecuciones extrajudiciales y desplazamiento forzado, entre otras graves violaciones de derechos humanos, ante la inminente entrada en vigor de un marco jurídico de amnistías a paramilitares durante el primer período presidencial de Álvaro Uribe Vélez.

El Movice se autodefine como "fruto de un extenso acumulado histórico de luchas de las víctimas por los derechos a la verdad, la justicia y la reparación integral en Colombia". ${ }^{1}$ A este movimiento pertenecen unas trescientas asociaciones civiles y grupos sociales y lo anteceden movimientos para la defensa de los derechos humanos y la sistematización de graves violaciones a los mismos en la búsqueda de conservar la verdad histórica.

Actualmente, en él confluyen diversas organizaciones y movimientos sociales, sindicales, políticos, étnicos y raciales que han sufrido la violencia generada por el Estado colombiano mediante graves violaciones a los derechos humanos, que se unieron con el propósito común de erradicar la impunidad.

El objetivo del Movimiento nacional de víctimas es lograr los derechos a la verdad, justicia, reparación integral, memoria y no repetición de los crímenes cometidos por el Estado colombiano, ${ }^{2}$ en torno al cual se han construido diversas estrategias para su reivindicación.

Alexander Herrera Varela, "Memoria colectiva y procesos de identidad social en el Movimiento de víctimas de crímenes de Estado" (Tesis de Maestría Universidad Nacional de Colombia, 2008), 13.

2 Movimiento nacional de víctimas de crímenes del Estado [Movice], "Las víctimas del conflicto armado en Colombia" http://datoscolombianuncamas.org/images/abook_file/INFORME\%20MOVICE\%20MAYO\%20 2010.pdf (acceso diciembre 26, 2016), 5. 
A partir de su surgimiento, este movimiento social ha elaborado su propia visión sobre la génesis y el sostenimiento de la violencia en Colombia y concibe que la mayor parte de la responsabilidad es atribuible al Estado colombiano, debido a que sus políticas en materia de "seguridad nacional" son propias de un Estado criminal en contra de la población civil, que ataca en especial a la sociedad civil organizada. ${ }^{3}$

Lemus resalta varios aspectos sobre algunas organizaciones que dieron origen al movimiento, como la Corporación Reiniciar y el Colectivo hijos e hijas por la memoria y contra la impunidad. En primer término se refiere a la evaluación del período de violencia en Colombia como un ciclo de crímenes de lesa humanidad que aún no termina y que debe ser recuperado por la memoria, misma que constituye un elemento novedoso para los movimientos sociales de la década del noventa.

El segundo aspecto se refiere a la relación con organizaciones internacionales, lo que hace del Movice un movimiento entallado en un ámbito regional, nacional e internacional. ${ }^{4}$

\subsection{El Movice y su constitución jurídica}

La constitución jurídica del Movimiento Nacional de Víctimas del Estado se llevó a cabo el 25 de junio de 2005 en Bogotá, donde se reunieron más de ochocientos delegados asistentes al II Encuentro nacional de víctimas de crímenes de lesa humanidad, violaciones graves a los derechos humanos y genocidio, quienes previo acuerdo y organización, dieron vida al Movice y definieron su identidad y sus propósitos. ${ }^{5}$

El Movimiento quedó compuesto por unas trescientas organizaciones de la sociedad civil $^{6}$ que son consideradas víctimas del Estado y están comprometidas con reivindicaciones que tienen que ver con la defensa de los derechos humanos. ${ }^{7}$ En el documento fundante del Movice se deja abierta la posibilidad de incorporar nuevas

3 Movimiento nacional de víctimas de crímenes del Estado [Movice], "La protección de las víctimas, ipaz con crímenes de Estado? La protección de las víctimas como imperativo para la paz, Informe, marzo 2015" http://www.movimientodevictimas.org/?q=content/informe-protecci\%C3\%B3n-de-las-v\%C3\%ADctimasde-cr\%C3\%ADmenes-de-estado-como-un-imperativo-para-la-paz (acceso diciembre 26, 2016), 16.

4 Giovanni Mora Lemus, "Memorias, pluralidad y movimiento social: la experiencia del Movice" (Tesis de Maestría, Pontificia Universidad Javeriana, 2010), 41.

5 Grupo de Memoria Histórica, Memorias en tiempo de guerra. Repertorio de iniciativas (Bogotá: Punto aparte, 2009), 185-188.

6 Grupo de Memoria Histórica, Memorias en tiempo de guerra, 188.

7 Herrera Varela, "Memoria colectiva y procesos de identidad social", 14. 
organizaciones cuando exista identidad de principios e iniciativas. Algunos de los movimientos sociales y las organizaciones no gubernamentales que lo conforman son los siguientes:

ONG's: Colectivo de abogados "José Alvear Restrepo", Comisión Intercongregacional de Justicia y Paz de la Conferencia de Religiosos de Colombia, Comité Permanente por la Defensa de los Derechos Humanos, Comisión de Justicia, Paz y Reverencia con la Creación de Familia Franciscana de Libertad CODEHSEL, Corporación Jurídica Libertad, Corporación Humanidad Vigente.

Presos Políticos: Fundación Comité de Solidaridad con los Presos Políticos FCSPP. Movimientos Sociales Cívicos: Comunidades Eclesiasticas de Mase Cébs, Misioneros Claretianos de Colombia, Proceso Comunidades Negras, Coordinadora Nacional de Afrodescendientes, Asociación Nacional de Ayuda Solidaria ANDAS, Instituto Nacional Sindical. Hijos y familiares de víctimas: Fundación Manuel Cepeda, Hijos e Hijas por la memoria, Unión Patriótica UP, Asociación de Familiares Detenidos-Desaparecidos —ASFADDES—, Asociación de Familiares de Víctimas de Trujillo. Campesinos: Asociación Nacional de Usuarios Campesinos ANUR-UR, Fensuagro, Coordinador Nacional Agrario, Federación Agrominera del Sur de Bolívar, Asociación de Campesinos de Antioquia, ADUC, Asociación Nacional de Desplazados. Sindicalistas: Central Unitaria de Trabajadores CUT, Unión Sindical Obrera USO Nacional, Instituto Nacional Sindical INS, Sindicato Nacional de Trabajadores de la Industria de Alimentos SINALTRAINAL, Sindicato de Trabajadores Mineros de Colombia SINTRAMINECOL, Asociación nacional de técnicos en telefonía y comunicaciones afines (ATELCA), ASODECOM, Asociación Distrital de Educadores ADE, Fenasintrap, Sintraelecol, Sintrateléfonos, Central Unitaria de Trabajadores. Indígenas: Comunidad de Kankuamos. Estudiantes: Asociación Colombiana de Estudiantes Universitarios. ${ }^{8}$

El Movimiento tiene su sede en Bogotá y, al estar conformado por víctimas de diversos lugares de Colombia, trabaja en una red de trece capítulos, correspondientes a las regiones de Bogotá, Antioquia, Atlántico, Atrato chocoano, Caquetá, Eje cafetero, Magdalena medio, Meta, Nariño, Putumayo, Sucre, Sur de Bolívar y Valle del Cauca. ${ }^{9}$

La amplitud y la riqueza del Movimiento no solo se refieren a la ubicación geográfica de sus miembros, sino también a la diversidad de organizaciones de defensa de

\footnotetext{
Herrera Varela, "Memoria colectiva y procesos de identidad social", 14

9 Grupo de Memoria Histórica, Memorias en tiempo de guerra, 189.
} 
derechos humanos de minorías o grupos en riesgo como los sindicalistas, indígenas, defensores de derechos humanos, estudiantes, campesinos, víctimas, presos políticos $\mathrm{y}$ afrodescendientes, entre otros.

\subsection{El surgimiento histórico del Movice}

El Movice surgió luego de varios acontecimientos encadenados y relacionados con la búsqueda de la reivindicación y organización de las víctimas, en respuesta a la impunidad de graves violaciones de derechos humanos causadas por el Estado colombiano.

Sus orígenes se remontan a la década del setenta, cuando aparecieron en Colombia los primeros abogados defensores de presos políticos y los grupos de mujeres que buscaban a sus esposos e hijos desaparecidos. ${ }^{10}$

En 1995 se propuso el proyecto Colombia nunca más, para resguardar información de graves violaciones a derechos humanos y crímenes de lesa humanidad por medio de la recolección y sistematización de información. Esta una base importante para la posterior constitución del movimiento, ${ }^{11}$ puesto que recogió los postulados éticopolíticos del proyecto. ${ }^{12}$

\subsubsection{El proyecto Colombia nunca más}

En Colombia se implementó una "guerra sucia" durante la década del ochenta como una estrategia de terror, cuyo fundamento fue la doctrina de la seguridad nacional importada de Estados Unidos y promovida desde la presidencia de Julio César Turbay Ayala. ${ }^{13}$

Ese contexto de violencia, dirigido en contra de actores sociales como sindicalistas, campesinos, estudiantes, indígenas, feministas, etc., originó diversas organizaciones

10 Movimiento nacional de víctimas de crímenes del Estado [Movice], "Las víctimas del conflicto armado en Colombia", 3.

11 Movimiento nacional de víctimas de crímenes del Estado [Movice], "Contra la impunidad y el olvido" en Debates de la memoria, aportes de las organizaciones de víctimas a una política de memoria, ed. Centro del bicentenario, memoria, paz y reconciliación (Bogotá: Alcaldía Mayor de Bogotá, 2008-2010), 38.

12 Herrera Varela, "Memoria colectiva y procesos de identidad social", 13.

13 Movimiento nacional de víctimas de crímenes del Estado [Movice], Paz sin crímenes de Estado. Memoria y propuestas de las víctimas (Bogotá: Autor, 2013), 11. 
de defensores de derechos humanos, en especial para los casos de torturas, desapariciones forzadas, masacres y ejecuciones extrajudiciales. ${ }^{14}$

Algo interesante de estos grupos defensores de derechos humanos es que mantenían una visión diferente de la violencia, pues consideraban al Estado como el principal violador de derechos humanos y concebían las medidas antisubversivas del Estado como desmesuradas y excesivas. ${ }^{15}$

Ya en la década del noventa, estas organizaciones se movilizaron con dos ideas claras: la superación de la impunidad y el castigo a los responsables de crímenes cometidos en la década anterior. ${ }^{16}$

A partir de entonces, las organizaciones y los movimientos sociales emplearon dos tácticas, que fueron la denuncia y la búsqueda de la solidaridad internacional, así como la utilización de mecanismos consignados en la Constitución de 1991. ${ }^{17}$

El proyecto Colombia nunca más tuvo como uno de sus factores de origen la campaña denominada "Colombia. Derechos humanos ya", impulsada por organizaciones sociales, grupos de derechos humanos y ONG a mediados de la década del noventa. En ella se hacía una denuncia nacional e internacional de las graves violaciones de derechos humanos, así como de la impunidad reinante en Colombia. ${ }^{18}$

El 10 de abril de 1995, diversas organizaciones no gubernamentales acordaron la realización del proyecto Colombia nunca más (PCNM), que comenzó con su primer equipo de trabajo en agosto de 1996 a registrar, documentar y sistematizar la información. ${ }^{19}$

Algunos aspectos que llevaron a dar un salto cualitativo a las estrategias de los defensores de derechos humanos para registrar y sistematizar estas violaciones fueron la promulgación de la Constitución de 1991 y la definición de crímenes de

\footnotetext{
14 Movimiento nacional de víctimas de crímenes del Estado [Movice], Paz sin crímenes de Estado, 12.

15 Movimiento nacional de víctimas de crímenes del Estado [Movice], Paz sin crímenes de Estado, 8.

16 Movimiento nacional de víctimas de crímenes del Estado [Movice], "Contra la impunidad y el olvido", 39.

17 Herrera Varela, "Memoria colectiva y procesos de identidad social", 9.

18 Grupo de Memoria Histórica, Memorias en tiempo de guerra, 175.

19 Centro de Memoria, Paz y Reconciliación, Rompecabezas de la memoria ¿Aportes de una comisión de la verdad? (Bogotá: Autor, 2014), 118;
} 
lesa humanidad en el Estatuto de Roma como un crimen generalizado o sistemático o ambos. $^{20}$

Lo anterior evidencia la evolución de las estrategias jurídicas de las víctimas ante la mayor disponibilidad de mecanismos constitucionales y la recepción del derecho sobre crímenes internacionales.

Sobre ello se produjo un debate muy interesante, ubicado en la interpretación de crímenes de lesa humanidad establecidos en el Artículo 7 del Estatuto de Roma. Por un lado, el Estado colombiano considera que son aquellos cometidos por las guerrillas y, por el otro, las víctimas del Estado y los defensores de derechos humanos los veían en aquellas violaciones cometidas por el Estado. ${ }^{21}$

Derivado del debate sobre la responsabilidad de crímenes de lesa humanidad en Colombia, las organizaciones defensoras de derechos humanos comenzaron a utilizar terminología especializada del derecho penal internacional al hablar de ataques generalizados y sistemáticos, para esquematizar las violaciones de derechos humanos cometidas por el Estado colombiano y mantener la verdad histórica.

El proyecto Colombia nunca más:

[... aspira a convertirse en la salvaguarda de la memoria colectiva, denuncia la impunidad como el engranaje principal que potencia la comisión [de crímenes de lesa humanidad] e identifica como ejes fundamentales el reclamo a la Verdad, la Justicia y la Reparación integral como articuladores exclusivos para superar el largo periodo de violencia vivido en Colombia. ${ }^{22}$

El proyecto definió sus propios objetivos como:

[...] un esfuerzo de un conjunto de organizaciones no gubernamentales, apoyada solidariamente por organizaciones también no gubernamentales de otros países, dirigido a salvaguardar la memoria de los crímenes de lesa

\footnotetext{
Corte Penal Internacional, Estatuto de Roma (Roma, 17 de julio de 1998), art. 7; Herrera Varela, "Memoria colectiva y procesos de identidad social", 9 .

21 Herrera Varela, "Memoria colectiva y procesos de identidad social", 9.

22 Herrera Varela, "Memoria colectiva y procesos de identidad social", 12.
} 
humanidad perpetrados en Colombia durante el último e inconcluso ciclo de violencia, que se inicia en $1965 .{ }^{23}$

El PCNM consideró la existencia de crímenes de Estado en Colombia como fruto de un terrorismo de Estado y no a causa de una dictadura militar y estableció que estos se perpetraron por omisión o con participación de agentes estatales independientemente o en colaboración con el paramilitarismo y de esta forma se cometieron desapariciones forzadas, torturas, asesinatos y masacres. ${ }^{24}$

Como complemento a esta tesis, el PCNM señaló que, con el afán de hacer frente a la subversión en Colombia, el Estado había ampliado su espectro de ataques a la población civil que se opone, discrepa o critica la política de seguridad del Estado, como fue el caso de la Unión Patriótica. ${ }^{25}$

Por su parte, el Grupo de Memoria Histórica expresa:

El Proyecto Colombia Nunca Más o PCNM es un proceso de construcción de memoria acerca de crímenes de Estado. Éste, en sus orígenes trabajó con un énfasis jurídico y, hoy articula estrategias de memoria activa en Internet, apoyadas en procesos de denuncia y pedagogía. ${ }^{26}$

El proyecto Colombia nunca más, al igual que el Movice, identifica varios períodos de violencia, en los que se han modificado los modelos de represión estatal. El período global analizado por ese proyecto fue desde 1965 hasta 1998.

Tabla 1. Períodos históricos de memoria del proyecto Colombia nunca más

\begin{tabular}{|c|l|}
\hline Períodos históricos de violencia & \multicolumn{1}{c|}{ Modelo de represión estatal } \\
\hline $1965-1981$ & La represión legal por medio del Estado de sitio \\
\hline $1982-1986$ & Represión paraestatal \\
\hline $1985-1990$ & Articulación entre represión legal y represión paraestatal \\
\hline $1991-1994$ & Expansión urbana del paramilitarismo \\
\hline $1994-1998$ & Modelo de legitimación y legalización del paramilitarismo \\
\hline
\end{tabular}

Fuente: Grupo de Memoria Histórica, Memorias en tiempo de guerra. Repertorio de iniciativas (Bogotá: Punto aparte, 2009), 181-184.

* Este cuadro ilustra la visión histórica de la violencia en Colombia del proyecto Colombia nunca más

\footnotetext{
23 Proyecto Colombia nunca más, Colombia, nunca más: crímenes de lesa humanidad (Bogotá: Autor, 2000), 3; Herrera Varela, "Memoria colectiva y procesos de identidad social", 11.

24 Grupo de Memoria Histórica, Memorias en tiempo de guerra, 176.

25 Grupo de Memoria Histórica, Memorias en tiempo de guerra, 176.

26 Grupo de Memoria Histórica, Memorias en tiempo de guerra, 175.
} 
Las organizaciones que conformaban el PCNM:

[... pretendían hacer una denuncia nacional e internacional de las múltiples y graves violaciones a los más elementales derechos y de la total impunidad en que se encontraban por la sistemática conducta del Estado a no hacer justicia, aun siendo el mayor agente responsable. ${ }^{27}$

Asimismo, tuvieron acercamientos con organizaciones internacionales para conocer las experiencias de comisiones de la verdad realizadas en diversos países de Sur y Centroamérica, lo que les permitió verificar que se trataba de instituciones parcialmente fallidas en la lucha contra la impunidad y les hizo ver la necesidad de documentar exhaustivamente los crímenes de lesa humanidad.

El PCNM documentó información de más de "4l.000 víctimas de torturas, desaparición forzada y/o ejecución extrajudicial en todo el país". ${ }^{28}$ Su trabajo constituyó un proyecto de memoria y se concentró en la investigación y sistematización de graves violaciones de derechos humanos y "de este proceso inicial surgió una conclusión contundente: la necesidad de una organización política de las víctimas, origen de la idea inicial del Movice". ${ }^{29}$

\subsection{Las reivindicaciones sociales del Movimiento nacional de víctimas de crímenes del Estado}

En una entrevista reciente, Camilo Villa, miembro del Movimiento nacional de víctimas, señaló que este movimiento tiene nueve estrategias para hacer realidad sus reivindicaciones sociales: ${ }^{30}$

1. Primera estrategia: jurídica, para castigar a los máximos responsables de los crímenes. Apunta a establecer la responsabilidad penal de agentes estatales por la ideación, planeación y ejecución de lo que ellos denominan crímenes de Estado.

${ }^{27}$ Proyecto Colombia nunca más, "Quiénes somos?" http://www.movimientodevictimas.org/ nuncamas/index. php?option=com_content\&view=article\&rid=1\&Itemid=310 (acceso diciembre 26, 2016).

28 Proyecto Colombia nunca más, "Quiénes somos?".

29 Movimiento nacional de víctimas de crímenes del Estado [Movice], "Contra la impunidad y el olvido", 42.

30 Movimiento nacional de víctimas de crímenes del Estado [Movice], "Las víctimas del conflicto armado en Colombia", 5-16. 
2. Segunda estrategia: audiencias ciudadanas por la verdad, una estrategia por la verdad y la memoria histórica. Pretende una sanción moral y pública de los responsables de los crímenes de Estado.

3. Tercera estrategia: comisión ética. Busca rechazar el olvido y dejar el mensaje de rechazo a la violencia como la forma de imponer un modelo político o social en Colombia.

4. Cuarta estrategia: prohibición constitucional del paramilitarismo. Tiene como fin constitucionalizar los derechos de las víctimas a la justicia, verdad y reparación, así como conseguir garantías de no repetición y prevención de estos crímenes atroces.

5. Quinta estrategia: el catastro alternativo. Su propósito es la restitución de tierras en un futuro, puesto que los campesinos desplazados forzadamente no tienen cómo acreditar su propiedad sobre las tierras y por eso se ha logrado usurpar la propiedad de miles de personas en Colombia.

6. Sexta estrategia: la exhumación de fosas comunes y el derecho a enterrar. Afirma el derecho a la verdad, el esclarecimiento de los hechos, la sanción penal de los responsables y la dignificación de las víctimas de desaparición forzada.

7. Séptima estrategia: lucha contra el genocidio político. Apunta a visibilizar y eliminar las prácticas genocidas contra grupos políticos alternativos.

8. Octava estrategia: organización y fortalecimiento del movimiento de víctimas. El Movice se reúne con diversas expresiones que han sido afectadas por la violencia del Estado para lograr una coordinación y consulta permanente con estas en el plano nacional e internacional.

9. Novena estrategia: retorno de los exiliados políticos. Se estudia el exilio de colombianos en el exterior y se buscan las garantías de no repetición y condiciones de dignidad para su retorno. 


\subsection{Colombia como Estado violento. La visión del conflicto de las víctimas del Estado}

El Movice expresa que la violencia en Colombia surgió en 1946 y se propagó hasta la actualidad. El Estado colombiano fue su mayor promotor y productor, puesto que se ha convertido en un Estado terrorista, que ha implementado diversas estrategias para la configuración de un plan o una política de graves crímenes en contra de la población. Este plan criminal se ha ejecutado de manera sostenida, gracias a la adopción de diversas estrategias estatales. ${ }^{31}$

\subsubsection{La adopción de una estrategia militar en perjuicio de una salida democrática}

El Estado colombiano tenía dos alternativas para hacer frente al levantamiento de armas de los grupos que buscaban cambios fundamentales en la política nacional: por un lado, un tratamiento político con un acercamiento para discusión de exigencias justas y, por el otro, la lucha armada para el exterminio de la subversión, que ha sido la estrategia adoptada, "pero no reduciendo la acción de exterminio a los alzados en armas sino llevándola a amplias capas de población civil desarmada, que caen o podrían caer bajo áreas de influjo territorial o ideológico de los combatientes". 32

\subsubsection{La adopción de la doctrina de lucha contrainsurgente para ejercer represión y control social}

Esta doctrina criminaliza de diversas formas cualquier manera de lucha por derechos, así como cualquier resistencia o disidencia y se concentra en atacar a sus dirigentes o líderes. ${ }^{33}$

\subsubsection{Adopción de estructuras permanentes de control social}

Se creó una estructura paramilitar por parte del Estado para la consecución y el mantenimiento del poder, que actúa con impunidad para atacar a la resistencia; para ello, se definieron marcos legales de persecución y castigo a la disidencia y para legitimar la represión por la fuerza pública.

\footnotetext{
Instituto Interamericano de Derechos Humanos, Módulos: regiones del Movimiento de víctimas de crímenes del Estado - Movice - (San José: Autor, 2011), 25-28.

32 Instituto Interamericano de Derechos Humanos, Módulos, 21.

33 Instituto Interamericano de Derechos Humanos, Módulos, 21.
} 
Lo más grave de esta estructura es que también considera la persecución y el ataque a civiles, más allá del combate con otros grupos armados, lo que incluye los movimientos sociales y la población.

Lo anterior requirió, por una parte, "la articulación operativa de lo militar con lo paramilitar, y por otra, la colaboración de los demás poderes mediante conductas omisivas, elusivas, encubridoras o desviacionistas. Sin la concurrencia de ambos polos, el modelo no funcionaría". ${ }^{44}$

\subsubsection{El terrorismo de Estado como método de control social}

Con este método se envían mensajes de miedo a la población para disuadir y desanimar a las organizaciones y los movimientos sociales en sus luchas. Se traducen en:

[...] acusaciones falsas, detenciones arbitrarias, montajes judiciales, juicios injustos, torturas, desapariciones forzadas, asesinatos individuales y colectivos, desplazamiento forzado de poblaciones, destrucción de bienes de subsistencia, bombardeos indiscriminados, amenazas, atentados y violencia sexual [...] estos métodos están encuadrados en una intención general de disuasión pues, como se ha visto, se trata de combatir y exterminar una forma de pensar, una ideología, una opción por un modelo alternativo de sociedad. ${ }^{35}$

El Movimiento de víctimas ha utilizado la definición de crímenes de Estado dada por J. Verhaegen, citada en un voto razonado por el juez de la Corte Interamericana Antonio Cancao Trindade, para dar un significado a las acciones criminales del Estado colombiano:

En los crímenes de Estado hay no sólo aquiescencia, sino también planificación por parte de las autoridades estatales, y acción ilícita por parte de múltiples perpetradores de violaciones graves de los derechos humanos y de sus colaboradores.

Instituto Interamericano de Derechos Humanos, Módulos, 27.

35 Movimiento nacional de víctimas de crímenes del Estado [Movice], "Nunca más", 26; Instituto Interamericano de Derechos Humanos, Módulos, 28. 
[Los crímenes de Estado son] ciertas prácticas sistemáticas de violaciones graves de los derechos humanos como parte de una política de Estado. ${ }^{36}$

El Movice argumenta que los crímenes de Estado están justificados a partir de la doctrina de la seguridad nacional y que los ataques son dirigidos en contra de sectores selectos, en general, aquellos que se encuentran en la lucha social, para presionarlos a que abandonen sus proyectos de vida. ${ }^{37}$

Las víctimas son aquellas que se relacionan con luchas por reivindicaciones sociales de diversos tipos, como campesinas, sindicales, políticas o comunitarias o que estén vinculadas con algunos sectores de la sociedad como indígenas, afrodescendientes, mujeres y otros. ${ }^{38}$

\subsubsection{La visión disidente del Movice sobre la violencia en Colombia}

La lectura que ha hecho el Movice sobre las causas y los responsables de la violencia en Colombia disiente de la sostenida por el Estado, pues este es señalado de ser su principal generador.

Existe por lo tanto una diferencia entre la versión oficial que explica la violencia en Colombia y la del Movice, pues esta última establece la falta de legitimidad democrática, ocasionada por la eliminación de contrincantes políticos, en contravía a la misma democracia.

La versión oficial asegura que la violencia se ejerce dentro del marco de la seguridad nacional que faculta el despliegue de acciones y justifica daños colaterales.

El Movice divide este gran período de 1946 hasta la actualidad en cinco fases más precisas en que se han adoptado diversas estrategias de violencia.

Corte Interamericana de Derechos Humanos, Caso Masacres de Ituango v. Colombia. Sentencia de xxxx, 1 de julio de 2006, voto razonado juez Antônio Cançado Trindade, párr. 22, 26.

37 Instituto Interamericano de Derechos Humanos, Módulos, 29.

38 Instituto Interamericano de Derechos Humanos, Módulos, 29. 
Tabla 2. Períodos de criminalidad estatal identificados por el Movice

\begin{tabular}{|l|c|}
\hline \multicolumn{1}{|c|}{ Fase } & Período de desarrollo \\
\hline Lucha entre liberales y conservadores. La Violencia. & $1946-1960$ \\
\hline Configuración del marco legal del paramilitarismo & $1962-1981$ \\
\hline Aparición pública, consolidación y expansión de estructuras paramilitares & $1981-1989$ \\
\hline $\begin{array}{l}\text { Pactos de paz en el marco de la nueva Constitución de 1991 y el nuevo fundamento } \\
\text { legal del paramilitarismo }\end{array}$ & $1990-2002$ \\
\hline Clímax del paramilitarismo & $2002-2010$ \\
\hline Criminalidad relacionada con la extracción de recursos por multinacionales & $2010-2014$ \\
\hline
\end{tabular}

Fuente: Movimiento nacional de víctimas de crímenes del Estado [Movice], Paz sin crímenes de Estado. Memoria y propuestas de las víctimas (Bogotá: Autor, 2013), 20-33.

\section{Teoría contemporánea de los movimientos sociales y el Movimiento nacional de víctimas de crímenes del Estado}

Los movimientos sociales han sido estudiados por la sociología desde tiempos recientes. En la década del sesenta entraron en vigor nuevas teorías, puesto que las anteriores no fueron capaces de cumplir más su cometido. Según la postura de algunos teóricos contemporáneos, existen diversos factores que nos ayudan a explicar el surgimiento y el éxito de un movimiento social y son los siguientes:

a) La estructura de oportunidades políticas.

b) La forma organizacional del movimiento

c) El proceso de encuadre de la realidad ejercida por el movimiento

d) El repertorio de acción colectiva utilizada (traducción propia). ${ }^{39}$

\subsection{La estructura de oportunidades políticas}

Para Tarrow, la estructura de oportunidades políticas puede definirse como "las dimensiones consistentes del entorno político que fomentan o desincentivan la acción

39 "1) the structure of political opportunities that confronts the movement; 2) the organisational form of the movement; 3) the process of framing' reality engaged in by the movement; and 4) the repertoire of collective action utilised by the movement". John C. Dugas, "The Origin, Impact and Demise of the 1989-1990 Colombian Student Movement: Insights from Social Movement Theory", Journal of Latin American Studies 33, núm. 4 (2001): 811. 
colectiva entre la gente" ${ }^{40}$ Según el autor, las oportunidades políticas surgen por factores externos al grupo, sobre todo por cambios en el Gobierno o en los grupos de élite en el poder que generan oportunidades para los ciudadanos comunes.

Entre los eventos que pueden generar oportunidades políticas se encuentran la apertura de acceso al poder, los cambios de alineamiento en el Gobierno, la disponibilidad de aliados con gran influencia y las divisiones entre élites. ${ }^{41}$

Las oportunidades políticas se pueden generar incluso por los mismos movimientos sociales, pues cuando uno de ellos aprovecha una y ejecuta una acción colectiva, se abren nuevas oportunidades para grupos afines, para la dialéctica entre movimientos y para movimientos contrarios.

Para Tarrow, los movimientos sociales están tan relacionados con oportunidades de carácter político, que incluso grupos débiles y desorganizados pueden sacar ventaja de una de ellas. La idea central de este postulado es que las personas se unen a los movimientos sociales como respuesta a una oportunidad política. ${ }^{42}$

\subsection{La forma organizacional del movimiento}

Para Dugas, existe un consenso entre los teóricos de los movimientos sociales sobre la importancia de la forma de organización de los movimientos para el éxito o fracaso de los mismos. ${ }^{43}$

En primer término, Tarrow señala que "un movimiento solo tendrá éxito cuando esté bien organizado". ${ }^{44}$ Considera que si bien es cierto que la determinación de participar en una acción colectiva es individual, quienes siempre activan y mantienen dicha acción son los grupos de contacto directo, las redes sociales y sus instituciones.

Por ello, los movimientos deben parecerse a una red de pequeños grupos, redes sociales y conexiones entre todos ellos: "[...] la movilización de redes sociales preexistentes reduce los costes sociales transaccionales de la convocatoria de

Sidney Tarrow, El poder en movimiento. Los movimientos sociales, la acción colectiva y politica (Madrid: Alianza, 1994), 49

41 Tarrow, El poder en movimiento, 49-50.

42 Tarrow, El poder en movimiento, 49.

43 Dugas, "The Origin, Impact and Demise", 812.

44 Dugas, "The Origin, Impact and Demise", 54. 
manifestaciones, y mantiene unidos a los participantes incluso una vez que el entusiasmo inicial de la confrontación se ha desvanecido". 45

Según Tarrow, los movimientos sociales van más allá de campañas aisladas, pues deben permanecer en unidad, así sea durante tiempos de aparente falta de efervescencia; "a menos que un movimiento mantenga su interacción con sus oponentes, sus aliados y las autoridades, es rápidamente ignorado y fácilmente reprimido". ${ }^{46}$

Para más claridad, Rucht ha clasificado los movimientos sociales junto con otros dos grupos sociales que pueden ser agentes de movilización, pero son más formales: los grupos de interés y los partidos políticos. ${ }^{47}$

\subsection{El proceso de encuadre de la realidad ejercida por el movimiento}

Dugas sostiene que el tercer factor que los teóricos encuentran decisivo para la suerte de los movimientos sociales es su capacidad de interpretar o enmarcar la realidad. ${ }^{48}$

Para el autor, el proceso de encuadre se define como "el esfuerzo consciente y estratégico realizado por grupos de personas para crear un entendimiento colectivo del mundo y de sí mismos para legitimar y motivar la acción colectiva". ${ }^{49}$

La tarea del encuadre de la realidad se refiere a tres tareas particulares: en primer lugar, generar encuadres dentro de los grupos y con la gente que han elegido como objetivo para obtener apoyo; se trata de que un mínimo de personas afectadas en algún aspecto de su vida puedan sentirse optimistas de que con la acción colectiva se solucionará el problema. ${ }^{50}$ En segundo lugar, un encuadre externo para hacer frente a las instituciones oficiales..$^{51}$ En tercer lugar, de nuevo se refiere a un aspecto interno de adaptación continua de los encuadres a la realidad para mantener la fuerza del grupo. ${ }^{52}$

45 Dugas, "The Origin, Impact and Demise", 56.

46 Dugas, "The Origin, Impact and Demise", 53.

47 Dieter Rucht, "El impacto de los contextos nacionales sobre la estructura de los movimientos sociales: un estudio comparado transnacional y entre movimientos" en Movimientos sociales perspectivas comparadas, coord. Doug McAdam, John McCarthy y Mayer Zald (Madrid: Itsmo, 1999), 265.

48 Dugas, "The Origin, Impact and Demise", 813.

49 Dugas, "The Origin, Impact and Demise", 813.

50 Dugas, "The Origin, Impact and Demise", 813-814.

51 Tarrow, El poder en movimiento, 123; Dugas, "The Origin, Impact and Demise", 814.

52 Dugas, "The Origin, Impact and Demise", 814. 


\subsection{El repertorio de acción colectiva}

Para Tilly, la acción colectiva es un concepto amplio - pero simple_-y la entiende como "aquello que incluye todas las maneras en que la gente une sus esfuerzos en la persecución de fines comunes". ${ }^{3}$

Dugas considera que el repertorio de acción colectiva "provee a los activistas de los movimientos sociales con una caja de herramientas, de acciones conocidas que pueden ser emprendidas para la persecución de reparación de agravios". ${ }^{54}$

La acción colectiva incluye una gran diversidad de comportamientos que van cambiando, pues cada grupo de personas con un objetivo común ha tenido a su alcance diversas medidas para ejecutar una acción colectiva, es decir, con el tiempo, se han modificado los repertorios. ${ }^{55}$

Tilly considera que las personas que tienen un interés común y deciden perseguirlo en colectivo tendrán un repertorio de acciones mayor o menor de acuerdo con los medios disponibles para su grupo, que sean útiles para enfrentar a las estructuras de poder. ${ }^{56}$

En las conclusiones de su estudio sobre los repertorios de acción colectiva, este autor considera que el estudio de la acción colectiva y sus repertorios nos permitirán hacer hallazgos políticos sobre las estructuras de poder que existen en determinado lugar y momento y, por ende, también comprenderemos qué estructuras contienden con aquellas.

\section{El Movimiento nacional de víctimas de crímenes del Estado frente a la teoría de los movimientos sociales}

A continuación se analiza el nacimiento y funcionamiento del Movimiento nacional de víctimas de crímenes del Estado a la luz de la teoría de los movimientos sociales contemporáneos, para determinar cuáles de ellos han sido cumplidos por el Movice y en qué medida se constituyó como una organización sólida.

53 Charles Tilly, "Studying Social Movements, Studying Collective Action", https://deepblue.lib.umich.edu/ bitstream/handle/2027.../168.pdf (acceso diciembre 26, 2016).

54 Dugas, "The Origin, Impact and Demise", 814.

55 Tilly, Studying Social Movements, 26-28.

56 Tilly, Studying Social Movements, 27. 


\subsection{La oportunidad política del Movimiento nacional de víctimas}

El proyecto Colombia nunca más comenzó a ejecutarse desde agosto de $1996^{57}$ y se planteó como una forma de memoria y sistematización de graves violaciones de derechos humanos cometidas en el país; sin embargo, hasta ese momento ni los defensores de derechos humanos ni las víctimas del Estado se habían organizado para rebasar la estrategia de producir memoria de los hechos.

Señala Camilo Villa que el Movimiento nacional de víctimas de crímenes del Estado se planificó como un movimiento a partir de la llegada de Álvaro Uribe Vélez a la Presidencia de la República en el año 2002, debido a las políticas antisubversivas y paramilitares que este encabezó en todo el país.

Destaca que, aunque ha existido paramilitarismo en Colombia desde hace mucho tiempo, fue durante el período presidencial de Álvaro Uribe que se practicó a la par de la promoción mediática de sentimientos "antiguerrilla" y de "pseudopatriotismo", en busca de la configuración de un marco legal para mantener en la impunidad los crímenes cometidos por paramilitares.

El Movice marca el año 2004 como un momento clave para el surgimiento de esta organización. Indica que:

[...] del 26 y 28 de agosto [de 2004] se realizó el encuentro Voces del mundo por Colombia, en el mismo instante en que el Ejecutivo tramaba la realización de un proyecto que diera la amnistía a grupos paramilitares, a partir de lo que se conoció como las negociaciones de Santafé de Ralito. ${ }^{58}$

Este proceso de construcción del Proyecto de ley 782 fue un golpe duro para las personas, las organizaciones no gubernamentales y los movimientos sociales que se consideraban víctimas y fue percibido como un ataque a su dignidad y como un beneficio a criminales que hicieron mucho daño a la sociedad colombiana.

Tarrow menciona que cuando se intenta responder a la pregunta sobre cuándo se pone en marcha un movimiento social, podemos también responder al por qué

Centro de Memoria, Paz y Reconciliación, Rompecabezas de la memoria, 117.

58 Movimiento nacional de víctimas de crímenes del Estado [Movice], "Contra la impunidad y el olvido", 43-44. 
sucede, ya que estos surgen gracias a oportunidades políticas, que en este caso fue el sentimiento de repudio ante el empuje de una ley de amnistía paramilitar.

La Ley 782 impulsada desde 2002 entró en colisión con la Corte Constitucional. Finalmente, luego de algunos cambios no sustanciales, se promulgó como la Ley 975 o Ley de justicia y paz, identificada por el Movice como una norma de impunidad.

En este contexto histórico surgió formalmente el Movimiento nacional de víctimas de crímenes del Estado el 25 de junio de 2005, en el seno del II Encuentro nacional de víctimas de crímenes de lesa humanidad, violaciones graves a los derechos humanos y genocidio.

\subsection{Apertura de acceso al poder}

Entre las oportunidades políticas que señala Tarrow encontramos la apertura de acceso al poder. A finales de la década del ochenta se promovió la redacción de una nueva Constitución que permitiera eliminar los cotos de poder encumbrados entre los Partidos Conservador y Liberal que se alternaban y coludían para el manejo del país, con la propuesta de la Séptima papeleta, impulsada por el Movimiento colombiano de estudiantes, que culminó con una nueva Constitución de 1991. ${ }^{59}$

Dentro del proceso constituyente se abrieron espacios importantes a actores sociales que no estaban acostumbrados a ser tomados en cuenta. La misma Asamblea Constituyente ya presentaba apertura a nuevos grupos:

La Asamblea Nacional Constituyente estuvo integrada por 23 miembros del Partido Liberal, 19 de la Alianza Democrática M-19, 11 del Movimiento de Salvación Nacional, 5 del Partido Conservador, 4 del Partido Conservador que se presentaron como independientes, 2 del Movimiento Unión Cristiana, 2 de la Unión Patriótica, 1 del Movimiento Unido Estudiantil, 1 de la Organización Nacional Indígena de Colombia, 1 de la Autoridades Indígenas de Colombia y 1 del Movimiento para un Nuevo País para los Niños. ${ }^{60}$

\footnotetext{
Dugas, "The Origin, Impact and Demise", 815.

60 Carmen Alicia Mestizo Castillo, "La gestación de la Constitución de 1991. El papel de la soberanía popular y debates en torno a ella" (Tesis de Maestría, Pontificia Universidad Javeriana, 2012), 105.
} 
La participación de diversos sectores en la redacción de la Constitución aseguró el ingreso de afrodescendientes y pueblos indígenas al Congreso; por ende, se configuró un acceso parcial a grupos que formarían parte del Movice.

Asimismo, se definieron mecanismos constitucionales como la acción de tutela y las acciones colectivas que permitían contar con más herramientas protectoras de derechos fundamentales y de participación ciudadana como el plebiscito y el referendo.

Según Lemus, "el proceso de la Asamblea Nacional Constituyente de 1991, es desde nuestro punto de vista un punto de quiebre, un antes y un después para los movimientos sociales en Colombia". ${ }^{61}$

\subsection{Cambios en las alineaciones del poder}

El surgimiento del Movice se presentó en el primer período presidencial de Álvaro Uribe Vélez (2002-2010) y, dado que no hubo alternancia en el Ejecutivo nacional, el Movimiento, en su inicio, no pudo aprovechar coyunturas políticas. La conformación del Senado y de la Cámara de Representantes contó con una mayor presencia de los Partidos Liberal y Conservador en las elecciones del año 2002 y los demás escaños quedaron polarizados entre una gran diversidad de partidos políticos.

\subsection{La disponibilidad de aliados con gran influencia}

El proyecto Colombia nunca más tuvo desde 1995 el apoyo de varios aliados que lograron el nacimiento y reforzamiento del Movice. Desde 1995, el proyecto Colombia nunca más tuvo el apoyo de diecisiete organizaciones no gubernamentales de derechos humanos y sociales que se sumaron al nacimiento y reforzamiento del Movice. Además, este proyecto compartía experiencias con otros proyectos Nunca más desarrollados en Argentina, Chile, Uruguay, Bolivia, El Salvador y Guatemala.

De acuerdo con el Movice, el nacimiento del proyecto Colombia nunca más se vio influenciado por una discusión simultánea en varios países de Centro y Suramérica sobre cómo hacer memoria histórica desde las víctimas; este debate transversal en el derecho internacional proponía la constitución de comisiones de la verdad.

${ }_{61}$ Mora Lemus, "Memorias, pluralidad y movimiento social", 14. 
La primera parte de nacimiento del Movice, que es el proyecto Colombia nunca más, encontró aliados en "otros procesos latinoamericanos", ${ }^{62}$ puesto que hubo "un proceso de acercamiento con las comisiones de verdad de Centro y Suramérica". ${ }^{63}$ Sin embargo, los más grandes adeptos que ha tenido son los movimientos, las asociaciones y las organizaciones que lo fundaron en el año 2005.

Además de las organizaciones y los movimientos, el Movice cuenta con adeptos en diversos medios de comunicación como Contagio Radio y Prensa Rural, pero no son aliados estratégicos. Estos últimos están en el ámbito político; ahí encontramos a Iván Cepeda Castro (uno de sus fundadores, representante a la Cámara 2010-2014, senador 2015-2019); Alirio Uribe Muñoz (miembro del Colectivo Alvear Restrepo, representante 2014-2017); Alexander López Maya (representante a la Cámara 2002-2005, senador 2006-2010 y 2010-2014); Alberto Castilla (líder agrario, senador 2014-2018) y Wilson Alfonso Borja Díaz (representante a la Cámara 2002-2005 y 2006-2009). Esto le ha permitido visibilizar sus reivindicaciones en el escenario nacional y exponer el tema de la violencia de Estado y sus víctimas, como lo hizo Iván Cepeda en septiembre de 2014 en un debate de control político ante el Senado de la República.

Los aliados del Movice en la Cámara de Representantes han logrado defender los derechos de las víctimas del Estado mediante la tramitación de iniciativas de reforma a la Constitución y a las leyes, con lo que se ha puesto en el centro de la mesa la agenda del Movimiento; prueba de ello es la iniciativa presentada por Wilson Alfonso Borja Díaz para elevar a rango constitucional los derechos de las víctimas de crímenes contra la humanidad y el establecimiento de mecanismos para garantizar la no repetición del paramilitarismo en Colombia (agosto 2007).El Movice ha creado nuevas sedes en el extranjero, como el capítulo Movice Valencia España (Movice_PV), creado en 2009 desde donde se siguen las labores de denuncia y visibilización del Movimiento y sus reivindicaciones en ese país, ${ }^{64}$ así como otras en México, Francia y Argentina.

Movimiento nacional de víctimas de crímenes del Estado [Movice], "Contra la impunidad y el olvido", 39. Movimiento nacional de víctimas de crímenes del Estado [Movice], "Contra la impunidad y el olvido", 40. 4 Movimiento nacional de víctimas de crímenes del Estado [Movice], "6 de marzo, Día internacional de las víctimas de crímenes del Estado colombiano" http://www.rebelion.org/noticia.php?id=181651 (acceso diciembre 26, 2016 


\subsection{Las divisiones entre élites}

Las élites no sufrieron una división considerable durante la época en la que surgió el Movimiento nacional de víctimas de crímenes del Estado; de hecho, fue un tiempo en el que estuvieron muy compactas, pues así se requería para el impulso y aprobación de la Ley de justicia y paz el 22 de junio de $2005 .{ }^{65}$

Luego de su presentación, el proyecto gubernamental de Ley de justicia y paz recibió críticas de parte de algunas ONG y una minoría de congresistas que señalaba su falta de mecanismos para garantizar los derechos de verdad, justicia y reparación que esta establecía.

Asimismo, se presentó un proyecto de ley alternativo que proponía una responsabilidad penal proporcional a los crímenes cometidos y era apoyado por organizaciones de víctimas, pero finalmente no tuvo éxito frente al proyecto gubernamental que, de acuerdo con Hernán Valencia, era "el fiel trasunto del proyecto político de 'seguridad democrática' de la administración del presidente Álvaro Uribe” y fue aprobado por el Congreso sin mayores contratiempos.

Así lo expresó Valencia Villa: "[... el el proceso ha sido conducido en forma solitaria por la cúpula del Ejecutivo, sin consulta con el órgano legislativo ni participación de la sociedad civil o de la comunidad internacional". ${ }^{66}$

El impulso de la Ley 975 de 2005 se debió a los congresistas más cercanos al Gobierno, ${ }^{67}$ con el uso de medios de comunicación masiva para generar consensos y aprobación. ${ }^{68}$ Fernanda Cárdenas y Hans Hartmann estudiaron el contexto de debate y aprobación de esa ley y revelaron un panorama negativo para una verdadera discusión informada. Afirman que las élites nacionales fueron la principal fuente de información de dichos medios, lo que generó una pobre discusión deliberativa.

Contrario a la conveniencia del Movice, en 2005 se descubrió un consenso de élites que facilitó un cubrimiento mediático unilateral y desequilibrado sobre la Ley de

\footnotetext{
65 Hernando Valencia Villa, La Ley de justicia y paz de Colombia a la luz del derecho internacional de los derechos humanos (Madrid: Fundación Hogar del Empleado, 2005), 5, 6 y 9.

66 Valencia Villa, La Ley de justicia y paz de Colombia, 6.

67 Rodrigo Uprimny, "Las leyes de justicia y paz en Colombia", http:// http://studylib.es/doc/95641/rodrigouprimny--las-leyes-de-justicia-y-paz-en-colombia (acceso diciembre 26, 2016), 6.

68 Valencia Villa, La Ley de justicia y paz de Colombia, 6.
} 
justicia y paz y que dejó fuera de las polémicas legislativas a los actores principales de la ley, que son las víctimas.

\begin{abstract}
El análisis de las condiciones estructurales sin embargo deja mucho que desear, pues las circunstancias en las que se realizan los debates sobre el conflicto armado y en especial el debate mediático sobre la Ley de Justicia y Paz hacen prever una baja calidad deliberativa de los MCM [medios de comunicación masiva]. Factores como el monopolio de la información oficial, un amplio consenso de élite, la autocensura y las condiciones de trabajo periodístico, así como las estructuras propietarias coartan la libertad de acción de los periodistas a la hora de escoger los actores presentes en el debate mediático y de examinar sus posiciones. ${ }^{69}$
\end{abstract}

Entonces, existió un consenso de las élites tanto en la estrategia mediática como legislativa, que culminó con la aprobación de la Ley de justicia y paz el 22 de junio de 2005.

\title{
3.6. La forma organizacional del Movimiento nacional de víctimas de crímenes del Estado
}

El Movimiento de víctimas se originó en la unión de organizaciones no gubernamentales, organizaciones de defensa de derechos humanos y movimientos sociales. Incluso su antecedente, el proyecto Colombia nunca más, provino de diecisiete organizaciones sociales y de derechos humanos, lo que deja ver que el Movimiento se derivó de la unión de organizaciones diversas preexistentes.

Al parecer, el Movice cumple con creces el estándar organizacional de Tarrow, pues está conformado por organizaciones preexistentes, lo que ayuda a reducir costes de convocatoria y le facilita mantenerse vigente aun en tiempos de ausencia de efervescencia; para la acción colectiva es importante la existencia de grupos de contacto directo y redes sociales que, en este caso, corresponde a un entramado de trescientas organizaciones de todo el país.

La pluralidad de miembros del Movimiento no ha sido problema para lograr una organización que le permita trabajar descentralizadamente por medio de cada uno

Julia María Fernanda Cárdenas y Jens Hartmann. "La Ley de justicia y paz en el diálogo público. La calidad deliberativa del discurso sobre la ley de justicia y paz en los medios masivos y su impacto en la aplicación de la misma", Criterio jurídico garantista 2, núm. 3 (2010): 172. 
de los capítulos regionales y centralizadamente mediante consultas hechas por la Secretaría Técnica Nacional a los capítulos. El Comité de impulso toma decisiones una vez que han sido comentadas con los capítulos y busca consenso para la posición que adoptará el Movice.

Cada seis meses se reúne el Comité de impulso ampliado, al que asisten delegados de los capítulos y cada dos años se realiza el encuentro anual, como un acto político en el que convergen víctimas de todo el país para exponer su postura sobre las víctimas de crímenes del Estado y la situación actual.

El manejo de las distancias y el consenso de las decisiones han permitido que el Movimiento mantenga la unidad y continúe con las actividades tendientes a ejecutar sus estrategias, con la diversidad como una riqueza y no como un lastre. ${ }^{70}$

\subsection{El proceso de encuadre de la realidad ejercida por el Movimiento}

El entendimiento colectivo que se generó con la creación del Movice para legitimar su acción colectiva es muy claro: se refiere a la adopción de la doctrina de la seguridad nacional y el terrorismo de Estado ejecutada por Colombia desde la década del cuarenta, que ha traído un sinfín de graves violaciones a derechos humanos y la comisión de crímenes de guerra y lesa humanidad. Desde la perspectiva de las víctimas, esta visión es el encuadre externo del movimiento.

Esta postura queda muy clara en diversos panfletos y manifiestos del Movimiento nacional, al identificarse como víctimas y señalar que el victimario es el Estado colombiano; un Estado terrorista que justifica sus crímenes con la doctrina importada de la seguridad nacional, consistente en:

[...] acusaciones falsas, detenciones arbitrarias, montajes judiciales, juicios injustos, torturas, desapariciones forzadas, asesinatos individuales y colectivos, desplazamiento forzado de poblaciones, destrucción de bienes de subsistencia, bombardeos indiscriminados, amenazas, atentados y violencia sexual [...], estos métodos están encuadrados en una intención general de DISUASIÓN, pues,

70 Movimiento nacional de víctimas de crímenes del Estado [Movice]. "Estructura organizativa". http://www. movimientodevictimas.org/?q=content/estructura-organizativa (acceso diciembre 26, 2016). 
como se ha visto, se trata de combatir y exterminar una forma de pensar, una ideología, una opción por un modelo alternativo de sociedad..$^{71}$

A pesar de que el Movimiento se compone de organizaciones y movimientos distintos, parece existir comunión de criterios sobre este punto y han construido una autoidentificación como víctimas que los une.

No obstante, el encuadre no solo es externo, sino que también debe desarrollarse desde el interior del propio movimiento, pues deben lograr que las personas que pertenecen a él se sientan agraviadas en algún aspecto de sus vidas y que al pertenecer al Movice están haciendo algo para cambiarlo colectivamente, esto es, mantener optimistas a sus miembros. ${ }^{72}$

Sobre este punto hay que resaltar que el Movice es consciente de que su lucha es contra un Estado armado, violento y con muchos recursos económicos y humanos nunca comparables con los del movimiento; en este sentido, el movimiento sabe de su posición y busca mantener la cohesión y unidad de sus miembros e incluso emplea una estrategia para permanecer organizados y fortalecidos frente a una tarea titánica.

\subsection{Repertorio de acción colectiva del Movimiento nacional de víctimas del Estado}

Tilly sostiene que los repertorios de acción colectiva se pueden entender como "la variedad de medios a través de los cuales gente ordinaria ha utilizado para actuar juntos". ${ }^{73}$ Estos medios han cambiado con el paso del tiempo y afirma que en la actualidad se puede hablar de algunos medios característicos como huelgas, intentos de revolución y reuniones masivas.

El Movimiento nacional de víctimas tiene un repertorio de acción colectiva muy amplio, que abarca las acciones consideradas como clásicas (marchas y huelgas, entre otras), pero me referiré a unas en especial que son las que me interesan en esta investigación: los litigios estratégicos ante tribunales internacionales (en particular, del Sistema Interamericano, Sistema Universal y de la Corte Penal Internacional) y

\footnotetext{
Movimiento nacional de víctimas de crímenes del Estado [Movice], "Nunca más", 26; Instituto Interamericano de Derechos Humanos, Módulos, 28.

72 Dugas, "The Origin, Impact and Demise", 813.

73 Tilly, Studying Social Movements, 14.
} 
la interacción que busca con organismos internacionales de vigilancia y monitoreo del respeto a los derechos humanos como la Comisión Interamericana de Derechos Humanos y algunas ONG de peso internacional como Human Right's Watch y Amnistía Internacional, para que visibilicen y se pronuncien sobre los asuntos que le conciernen al Movimiento.

El desarrollo de investigaciones sociojurídicas y la elaboración de informes y publicaciones también son actividades importantes que le permiten al Movimiento difundir su visión integral del fenómeno de la violencia generada por el Estado desde la perspectiva de las víctimas y posicionarse públicamente.

Una cualidad muy importante para el desarrollo de las estrategias del Movice es contar con organizaciones de defensores de derechos humanos que hacen uso del litigio estratégico para la visibilización de graves violaciones y el reconocimiento y goce de derechos humanos de las víctimas, como la Fundación comité de solidaridad con los presos políticos, el Colectivo de abogados José Alvear Restrepo (Cajar) y el Comité permanente por la defensa de los derechos humanos.

Además, el Movimiento tiene estrategias importantes e innovadoras para la memoria y la reparación integral como el registro catastral alterno al del Estado colombiano, en busca de recuperar la situación anterior al desplazamiento de miles de víctimas, la construcción de memoria y verdad mediante audiencias ciudadanas, la exhumación de fosas comunes y la promoción del derecho a enterrar a víctimas.

\section{Conclusiones}

El proyecto Colombia nunca más, en el que participaban diversos movimientos sociales y organizaciones no gubernamentales, fue el antecedente más importante del Movice, del cual heredó la sistematización de graves violaciones de derechos humanos, la construcción de memoria histórica y la lucha contra la impunidad.

De acuerdo con los parámetros de las teorías contemporáneas de los movimientos sociales, se interpreta que el Movice surgió de la posibilidad de la entrada en vigor del marco jurídico de Justicia y paz, que otorgaba demasiadas concesiones a los paramilitares responsables de graves violaciones de derechos humanos; ello generó la efervescencia de varios movimientos sociales, organizaciones no gubernamentales y otros grupos que decidieron unirse para hacer frente desde un solo movimiento a este conjunto de amnistías. En resumen, esta fue la oportunidad política con la 
que muchos se sintieron identificados y fue aprovechada para conglomerar unas trescientas organizaciones de todo el país.

Los aliados estratégicos del Movimiento han sido en realidad sus numerosos miembros, lo que le otorga una gran diversidad y le facilita hacer presencia en gran parte del territorio nacional y extranjero, para visibilizar sus acciones y sus exigencias. Algunos de sus miembros han logrado llegar a la Cámara de Representantes y al Senado, con lo que acerca los derechos de las víctimas al debate público; además, tiene como aliados a diversos medios de comunicación que comulgan con la protesta contra la violencia estatal en Colombia.

La organización del Movimiento y su conformación diversa han fortalecido su permanencia, con base en redes sociales sólidas. Asimismo, su estructura le facilita trabajar descentralizadamente en los territorios de cada capítulo y centralizadamente, con encuentros semestrales y anuales, con lo que ha hecho presencia en gran parte del territorio nacional.

El proceso del encuadre de la realidad le ha permitido al Movimiento contar con una postura propia sobre el fenómeno de la violencia en Colombia, en la cual se subraya la gran responsabilidad del Estado.

El repertorio de acción colectiva del Movice abarca estrategias que son innovadoras y dan respuesta al mantenimiento de la memoria y a la consecución de la justicia y la verdad. Entre ellas se encuentran algunas acciones clásicas y un mecanismo jurídico que incluye el litigio estratégico ante tribunales internacionales. Cabe destacar que con las investigaciones y los informes, el Movimiento fija su postura histórica y política sobre el conflicto, en contienda con la concepción estatal de los derechos humanos y de la violencia.

Para finalizar, considero que con base en los elementos explicativos del surgimiento de los movimientos sociales, el Movice cumple con una forma de organización y conformación, así como con un importante encuadre de la realidad que no solo le sirve para mantener la efervescencia y el interés entre sus agremiados, sino para poner en el plano social una postura propia que legitime y respalde sus exigencias sociales frente al Estado. 


\section{Referencias}

Cárdenas, Julia María Fernanda y Jens Hartmann. "La Ley de justicia y paz en el diálogo público. La calidad deliberativa del discurso sobre la ley de justicia y paz en los medios masivos y su impacto en la aplicación de la misma". Criterio jurídico garantista 2, núm. 3 (2010): 158-172.

Centro de Memoria, Paz y Reconciliación. Rompecabezas de la memoria iAportes de una comisión de la verdad? Bogotá: Autor, 2014.

Corte Interamericana de Derechos Humanos. Caso Masacres de Ituango v. Colombia. Sentencia de x xxx, 1 de julio de 2006. Voto razonado juez Antônio Cançado Trindade.

Corte Penal Internacional. Estatuto de Roma. Roma, 17 de julio de 1998.

Dugas, John C. "The Origin, Impact and Demise of the 1989-1990 Colombian Student Movement: Insights from Social Movement Theory". Journal of Latin American Studies 33, núm. 4 (2001): 807-837.

Grupo de Memoria Histórica. Memorias en tiempo de guerra. Repertorio de iniciativas. Bogotá: Punto aparte, 2009.

Herrera Varela, Alexander. "Memoria colectiva y procesos de identidad social en el Movimiento de víctimas de crímenes de Estado". Tesis de Maestría, Universidad Nacional de Colombia, 2008.

Instituto Interamericano de Derechos Humanos. Módulos: regiones del Movimiento de víctimas de crimenes del Estado - Movice-. San José: Autor, 2011.

Mestizo Castillo, Carmen Alicia. "La gestación de la Constitución de 1991. El papel de la soberanía popular y debates en torno a ella”. Tesis de Maestría, Pontificia Universidad Javeriana, 2012.

Mora Lemus, Giovanni. "Memorias, pluralidad y movimiento social: la experiencia del Movice". Tesis de Maestría, Pontificia Universidad Javeriana, 2010.

Movimiento nacional de víctimas de crímenes del Estado [Movice]. "Contra la impunidad y el olvido" en Debates de la memoria, aportes de las organizaciones de víctimas a una política de memoria, editado por Centro del bicentenario, memoria, paz y reconciliación, 37-50. Bogotá: Alcaldía Mayor de Bogotá, 2008-2010.

Movimiento nacional de víctimas de crímenes del Estado [Movice]. "Las víctimas del conflicto armado en Colombia". http://datoscolombianuncamas.org/images/abook_file/ INFORME\%20Movice\%20MAYO\%202010.pdf (acceso diciembre 26, 2016).

Movimiento nacional de víctimas de crímenes del Estado [Movice]. Paz sin crímenes de Estado. Memoria y propuestas de las víctimas. Bogotá: Autor, 2013

Movimiento nacional de víctimas de crímenes del Estado [Movice]. "Nunca más". https:// www.iidh.ed.cr/IIDH/media/1586/modulos-2010.pdf (acceso diciembre 26, 2016). 
Movimiento nacional de víctimas de crímenes del Estado [Movice]. La protección de las víctimas, ¿paz con crímenes de Estado? La protección de las víctimas como imperativo para la paz, Informe, marzo 2015. http://www.movimientodevictimas.org/?q=content/ informe-protecci\%C3\%B3n-de-las-v\%C3\%ADctimas-de-cr\%C3\%ADmenes-deestado-como-un-imperativo-para-la-paz (acceso diciembre 26, 2016).

Movimiento nacional de víctimas de crímenes del Estado [Movice]. "6 de marzo, Día internacional de las víctimas de crímenes del Estado colombiano". http://www. rebelion.org/noticia.php?id=181651 (acceso diciembre 26, 2016).

Movimiento nacional de víctimas de crímenes del Estado [Movice]. "Estructura organizativa". http://www.movimientodevictimas.org/?q=content/estructura-organizativa (acceso diciembre 26, 2016).

Proyecto Colombia nunca más. "Quiénes somos?". http://www.movimientodevictimas. org/ nuncamas/index.php?option=com_content $\&$ view=article $\&$ rid $=1 \&$ Itemid $=310$ (acceso diciembre 26, 2016).

Proyecto Colombia nunca más. Colombia, nunca más: crímenes de lesa humanidad. Bogotá: Autor, 2000.

Rucht, Dieter. "El impacto de los contextos nacionales sobre la estructura de los movimientos sociales: un estudio comparado transnacional y entre movimientos" en Movimientos sociales perspectivas comparadas, coordinado por Doug McAdam, John McCarthy y Mayer Zald, 262-287. Madrid: Itsmo, 1999.

Tarrow, Sidney. El poder en movimiento. Los movimientos sociales, la acción colectiva y política. Madrid: Alianza, 1994.

Tilly, Charles. "Studying Social Movements, Studying Collective Action". https://deepblue. lib.umich.edu/bitstream/handle/2027.../168.pdf (acceso diciembre 26, 2016)

Uprimny, Rodrigo. "Las leyes de justicia y paz en Colombia". http:// http://studylib. es/doc/95641/rodrigo-uprimny--las-leyes-de-justicia-y-paz-en-colombia (acceso diciembre 26, 2016).

Valencia Villa, Hernando. La ley de Justicia y Paz de Colombia a la luz del Derecho Internacional de los derechos humanos. Madrid: Fundación Hogar del Empleado, 2005. 